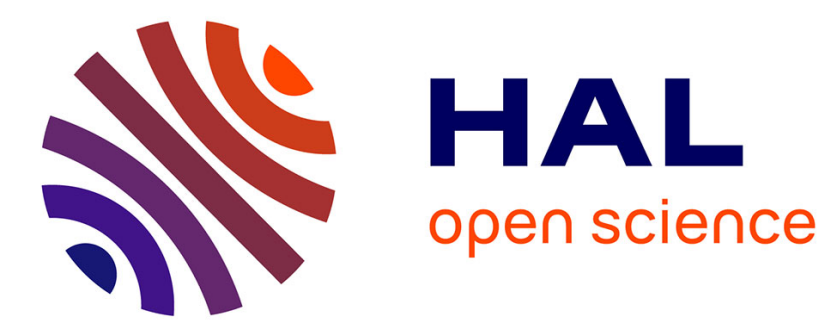

\title{
Modeling the Polarization Ratio in the Upper Microwave Band for Sea Clutter Analysis
}

Floriane Madeleine Schreiber, Sebastien Angelliaume, Charles-Antoine Guérin

\section{To cite this version:}

Floriane Madeleine Schreiber, Sebastien Angelliaume, Charles-Antoine Guérin. Modeling the Polarization Ratio in the Upper Microwave Band for Sea Clutter Analysis. IEEE Transactions on Geoscience and Remote Sensing, In press, pp.1-13. 10.1109/TGRS.2020.3025683 . hal-02960577

\section{HAL Id: hal-02960577 \\ https://hal.science/hal-02960577}

Submitted on 25 May 2021

HAL is a multi-disciplinary open access archive for the deposit and dissemination of scientific research documents, whether they are published or not. The documents may come from teaching and research institutions in France or abroad, or from public or private research centers.
L'archive ouverte pluridisciplinaire HAL, est destinée au dépôt et à la diffusion de documents scientifiques de niveau recherche, publiés ou non, émanant des établissements d'enseignement et de recherche français ou étrangers, des laboratoires publics ou privés. 


\title{
Modelling the Polarization Ratio in the upper microwave band for Sea Clutter Analysis
}

\author{
Floriane Madeleine SCHREIBER ${ }^{1,2}$, Sébastien ANGELLIAUME ${ }^{1}$, Charles-Antoine GUERIN ${ }^{2}$ \\ ${ }^{1}$ DEMR, ONERA, F-13661 Salon cedex Air - France \\ ${ }^{2}$ Université de Toulon, Aix Marseille Université, CNRS, IRD, MIO UM 110, La Garde, France
}

\begin{abstract}
The Two-scale Model (TSM) based on Bragg tilting is widely employed to model the normalized radar cross section (NRCS) from the sea surface in the microwave bands. It also leads to the compound distribution model when used to represent the distribution of intensity in high-resolution sea clutter. While the TSM satisfactorily describes the dynamical behavior of the polarized NRCS at moderate incidence angles for various sea states and radar freqencies, it fails to consistenly describe the polarized sea clutter distribution in the upper microwave band. The hidden reason is an overestimation of the Bragg polarization ratio (PR) at larger local incidence angles. Following the concept of universal analytical scattering models and based on the experimental evidence of several independent $X$ band airborne datasets, we propose a simple correction to the facet NRCS which brings the PR in closer agreement to the observations and makes it analytically very close to the popular Thompson model. This amended model preserves the simple structure of Bragg scattering; it admits one single extra parameter which makes a dynamical transition between the asymptotic Bragg and Kirchhoff regime depending on the sea state and the radar frequency. This allows to correctly describe the angular variations of the $P R$ in the upper microwave regime $(\mathrm{C}, \mathrm{X}$ and $\mathrm{Ku}$ bands) with minimal a priori information regarding the sea surface. Once this correction is incorporated in the TSM, a consistent modelling of the polarized sea clutter intensity can be obtained with the compound distribution model.
\end{abstract}

\section{INTRODUCTION}

Radar remote sensing is a unique tool for global ocean surface monitoring in many civil applications such as ship detection, search and rescue operations, etc. For these tasks which are related to target identification in a complex environment, sea clutter discrimination is a key issue. With an imaging radar operating at low spatial resolution the pixel intensity, which is proportional to the backscattered sea surface signal on each radar cell, is known to follow an exponential probability distribution; if one considers the signal amplitude rather than the signal intensity as it is often the case, this corresponds to a Rayleigh distribution. However, as the resolution increases, the backscattered intensity of sea clutter deviates from this distribution and is better represented by heavy tail distributions such as Pareto, Weibull or K distributions [1], [2], [3], [4], [5]. Now, even though these distributions provide consistent fits with the observations using a limited number of tuning parameters (typically, the "shape" and "scale" parameter), they are purely empirical and cannot be used for a systematic inversion of the sea state parameters. Nevertheless, there are both experimental evidences and theoretical arguments to support the fact that the actual intensity distribution depends on the oceanic parameters and that increasing sea state enhances the departure from the exponential distribution. Valenzuela [6] was the first to use the idea of a compound probability distribution based on a TwoScale Model (TSM) to account for the non-Rayleigh statistics of sea clutter amplitude. He attributed the latter to the modulation of the pixel amplitude (the so-called texture) by the tilting effect of long waves. This idea was subsequently pursued by other authors; in particular [7] showed that, assuming a perfectly conducting surface, the compound model could explain analytically the lognormal shape of the sea clutter amplitude distribution in the horizontal polarization. The primary conclusions of [6] were recently confirmed by the theoretical analysis and the realistic numerical simulations presented in [8]; in this work, an improved TSM was used, where smallscale diffraction was treated by the second-order SmallSlope Approximation in order to also investigate the bistatic configuration and the cross-polarized components.

\section{THE COMPOUND DISTRIBUTION MODEL}

In high-resolution radar imaging the pixel size is typically of the order of a few radar wavelengths. It is therefore natural to describe the radar reflectivity in terms of a Two-Scale Model (TSM, [9], [10], [11]) in which the elementary rough facets coincide with image pixels. In this model the Normalized Radar Cross Section (NRCS) from the sea surface is obtained by an incoher- 
ent average of the rough facet backscattered intensity according to the facet slope distribution $(\operatorname{pdf}(s))$ :

$$
\sigma^{0}(\theta)=\int \operatorname{pdf}(s) \sigma_{L}^{0}(\theta ; s) d s,
$$

where $\sigma_{L}^{0}(\theta ; s)$ is the NRCS expressed in the local frame of reference attached to one facet but projected onto the polarization basis attached to the global frame of reference. For a single look image, the fluctuations of pixel intensity $I$ (the speckle) are exponentially distributed about a mean quantity $\sigma_{L}^{0}(\theta ; s)$ referred to as the texture, which is the local NRCS attributed to a tilted facet of size $L$ and slope $s$. The conditional probability distribution function (pdf) of facet intensity for a given facet slope vector $s$ is therefore:

$$
\operatorname{pdf}(I \mid s)=\frac{1}{\sigma_{L}^{0}(\theta ; \boldsymbol{s})} \exp \left(-\frac{I}{\sigma_{L}^{0}(\theta ; \boldsymbol{s})}\right), u \geq 0,
$$

where $\theta$ is the incidence angle. The unconditional pdf of facet intensity is obtained by further integration over the facet slope distribution:

$$
\operatorname{pdf}(I)=\int \operatorname{pdf}(I \mid s) \operatorname{pdf}(s) d s
$$

Note that due to facet tilting the local incidence angle can exceed 90 degrees. In that case, the corresponding local NRCS is set to zero (or to the instrument noise floor in case of experimental data) to ignore this effect. It is often advantageous to express the clutter distribution in terms of a dimensionless intensity $\tilde{I}$ such that $I=\sigma^{0}(\theta) \tilde{I}$, where $\sigma^{0}(\theta)$ is the NRCS of the infinite surface, assumed to be statistically homogeneous. The resulting compound distribution for the normalized intensity is given by:

$$
\operatorname{pdf}(\tilde{I})=\int \frac{1}{\mathcal{T}(\theta ; \boldsymbol{s})} \exp \left(-\frac{\tilde{I}}{\mathcal{T}(\theta ; \boldsymbol{s})}\right) \operatorname{pdf}(\boldsymbol{s}) d \boldsymbol{s},
$$

where $\mathcal{T}(\theta ; s)=\sigma_{L}^{0}(\theta ; s) / \sigma^{0}(\theta)$ is a dimensionless quantity which we refer to as the "texture" parameter. In the absence of long wave tilt this parameter reduces to unity and the exponential distribution is recovered in (4). On the contrary, the occurrence of strong facet slope increases the deviation from the latter. The facet slope distribution is usually assumed to be a two-dimensional Gaussian, discarding skewness and peakedness effects as well as extreme slopes related to breaking events.

\section{The "Bragg Family" of Two-Scale Models}

The classical way to describe scattering by small-scale roughness within the TSM is the Small-Perturbation Method ([12]), also known as Bragg scattering model in the oceanic context (henceforth simply referred to as the "Bragg model"). At first-order in roughness, the copolarized components of the NRCS are given by [10]:

$$
\sigma^{0}(\theta)=16 \pi K_{0}^{4} \cos ^{4} \theta \mathcal{B}(\theta) S\left(\boldsymbol{q}_{H}\right)
$$

where $K_{0}=2 \pi / \lambda$ is the radar wave number and $\theta$ is the incidence angle, $S$ is the two-dimensional wave number spectral density of ocean roughness (with normalisation $\left.\int d \boldsymbol{k} S(\boldsymbol{k})=\left\langle\eta^{2}\right\rangle\right)$ and $\mathcal{B}$ is the first-order Bragg scattering kernel depending on the incidence angle, sea surface relative permittivity $\epsilon$ and the polarization. The ocean surface spectrum $S$ is only involved through a single wave number $\boldsymbol{q}_{H}$, referred to as the Bragg resonant wave vector and equal to twice the horizontal projection of the incident wave vector (so that $q_{H}=2 K_{0} \sin \theta$ in norm).

When used to describe the local NRCS of a rough facet in its local frame of reference it takes the form:

$$
\sigma_{L}^{0}(\theta ; s)=16 \pi K_{0}^{4} \cos ^{4} \theta_{s} \mathcal{B}_{s} S\left(\boldsymbol{q}_{H, s}\right)
$$

where $s$ is the facet slope, $\theta_{s}$ and $\boldsymbol{q}_{H, s}$ are the local incidence angle and Bragg resonant wave vector attached to this facet, respectively, and $\mathcal{B}_{s}$ is the tilted Bragg kernel obtained by projection of the horizontally and vertically components of the Bragg kernel onto the polarization basis (see eq. (5.1)\&(5.2) in [10]). For small slopes, the effect of the out-of-plane tilting (that is, perpendicular to the plane of incidence) is negligible and the local Bragg kernel along the vertical or horizontal polarization is essentially given by expressing the Bragg kernel at the local incidence angle, $\mathcal{B}_{s} \simeq \mathcal{B}\left(\theta_{s}\right)$. Hence, the distribution of facet NRCS depends primarily of the mean-square slope (mss) in the radar look direction $\left(\operatorname{mss}_{x}\right)$ and is quasi-insensitive to the radar cross-look direction $\left(\operatorname{mss}_{y}\right)$.

The TSM based on Bragg tilting (henceforth referred to as the "tilted Bragg model") has been for long the most popular model for ocean scattering [10] and has been shown to give correct qualitative trends of ocean backscatter and fair quantitative agreement with experimental data at medium incidences, especially in the lower part of the microwave frequency band ( $\mathrm{L}$ or $\mathrm{S}$ band). In particular, it is able to provide a (slight) dependence on roughness of the polarization ratio contrarily to the original Bragg scattering model. Another interesting feature of the tilted Bragg model in ocean backscattering modelling is that it involves only a limited knowledge of the sea surface spectrum, namely a small wave number interval around the Bragg resonant frequency (due to the variation of the local incidence angle) and is simple to implement. However, the tilted Bragg model has some acknowledged limitations; it is dependent on the facet size, which makes the latter choice somewhat arbitrary, 
and it fails at low incidence angles. In this respect, the use of the first-order Bragg model for the local facet cross section can be advantagously replaced by the use of the first-order Small-Slope Approximation (SSA, [13]), leading to the cut-off invariant Two-Scale Model [11], henceforth simply referred to as GO-SSA. This last model has a wider angular domain of validity as it holds at low incidence angles and is robust to the choice of the cut-off scale. Nevertheless, these 3 models from the "Bragg family" (Bragg model, tilted Bragg model, GO-SSA) yield close results when employed at midincidence for co-polarized components.

An important parameter when assessing the accuracy of a scattering model is the Polarization Ratio (PR), defined as the ratio of the Vertically to Horizontally copolarized NRCS ( $\left.P R=\sigma_{V V}^{0} / \sigma_{H H}^{0}\right)$. It turns out, that the PRs predicted by models of the "Bragg family" are way too large when applied at mid- or large incidence and compared to data acquired in the upper part of the microwave frequency band $(\mathrm{C}, \mathrm{X}, \mathrm{Ku}, \mathrm{Ka})$. Figure 1 shows the PR as a function of the incidence angle calculated with an omnidirectional Elfouhaily spectrum [14] according to the 3 models of the "Bragg family" and compared with experimental $\mathrm{X}$ band data from the POLLUPROOF [] and INGARA [15] datasets. Even the more accurate model (GO-SSA) overestimates the PR by at least $2 \mathrm{~dB}$ at 50 degree incidence.

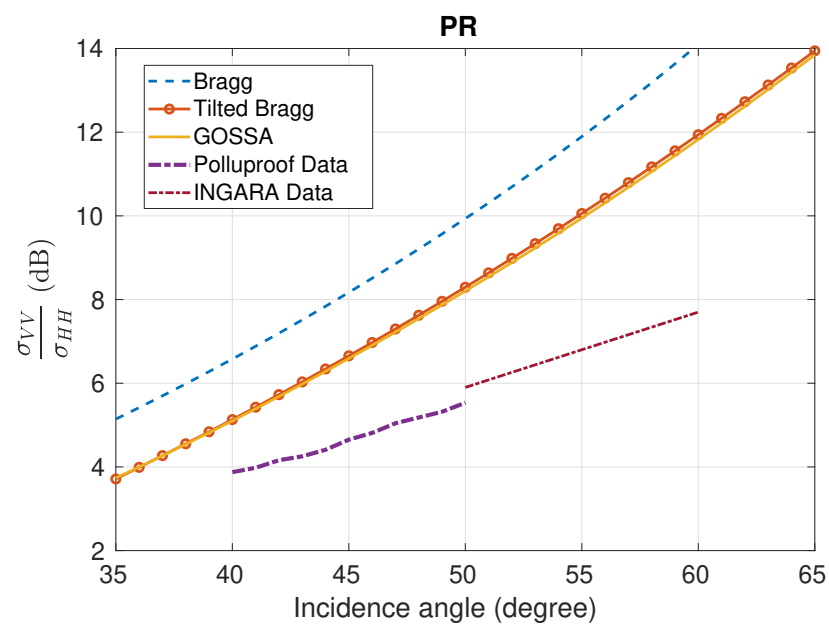

Fig. 1: $X$ band polarization ratio (in $\mathrm{dB}$ ) as a function of the incidence angle according to several models from the "Bragg family" (Bragg model, tilted-Bragg model and GO-SSA1) and comparison with experimental data sets for a wind speed about $10 \mathrm{~m} / \mathrm{s}$.

This effect is systematic and does not depend on the choice of the ocean spectrum nor on the wind direction. We have tested various spectral models at several wind speed: the omnidirectional and directional Elfouhaily spectrum, the omnidirectional Kudryavtsev spectrum
[14] and the omnidirectional Bringer spectrum [16], with virtually no difference for the resulting PR.This quasi insensibility of the PR to the spectral model is plain from the expression of the tilted Bragg model since the NRCS is proportional to the wave spectrum in the vicinity of the Bragg frequency. The same is verified for the GOSSA model which is very close to the tilted Bragg model at medium incidences. This few $\mathrm{dB}$ observed difference might be acceptable when trying to model the large dynamics of the co-polarized NRCS over a large span of incidence angles and sea states. However, having an accurate PR is crucial when it comes to the modelling of sea clutter intensity distribution. The reason is, the distribution of facet intensity is primarily accounted for by the sensibility of the local NRCS to the tilt angle. Whereas in the global NRCS the main contributing facets are those with small and positive slopes, the entire set of facet slopes must be taken into account to model the distribution of intensity. Hence, a good estimation of the total NRCS can be obtained with less stringent conditions on the PR which need only be accurate at small and moderate incidence angles. On the contrary, the PR must remain accurate over a large range of incidence to allow for a correct modelling of the clutter distribution. To support this claim, we calculated the distribution of normalized intensity (eq. (4)) according to the tilted Bragg model using different realistic values of the $\operatorname{mss}_{x}$ in the radar look direction (as already stated, the effect of the cross-slope $\operatorname{mss}_{y}$ is negligible). It turns out that the experimental intensity distributions obtained in each co-polarization chanel (VV or $\mathrm{HH}$ ) are inconsistent with the tilted Bragg model as they cannot be fitted simultaneously using the same value of $\operatorname{mss}_{x}$. It takes a larger value $\left(\operatorname{mss}_{x}=0.02\right)$ to match the VV data than the HH data $\left(\operatorname{mss}_{x}=0.008\right)$, as seen in Figure 2. The higher value of mss needed to fit the VV data in the tilted Bragg model can be explained by either the insufficient sensitivity of the VV Bragg kernel or the exagerated sensitivity of the $\mathrm{HH}$ Bragg kernel to the tilt angle. This is seen in Figure 3, where the geomretrical factor $\cos ^{4} \theta \mathcal{B}(\theta)$ of the Bragg model is plotted as a function of the incidence angel for the two polarizations. While the $\mathrm{HH}$ factor can vary by about $20 \mathrm{~dB}$ when the local incidence angle changes from 0 to 60 degrees, the VV undergoes only weak variations (less than $3 \mathrm{~dB}$ over the same angular domain). Therefore, it seems that the Bragg kernels do not have the appropriate dependence to the local incidence angle to account for the distribution of facet intensities. 

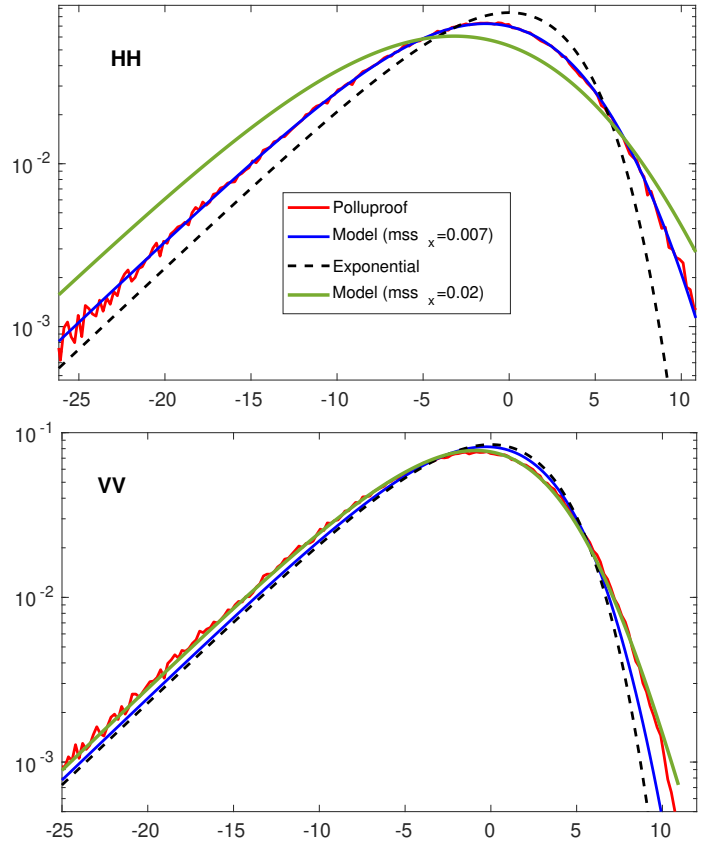

Fig. 2: Intensity distribution for the VV and $\mathrm{HH}$ clutter at 45 degree incidence for different values of $\operatorname{mss}_{x}$.

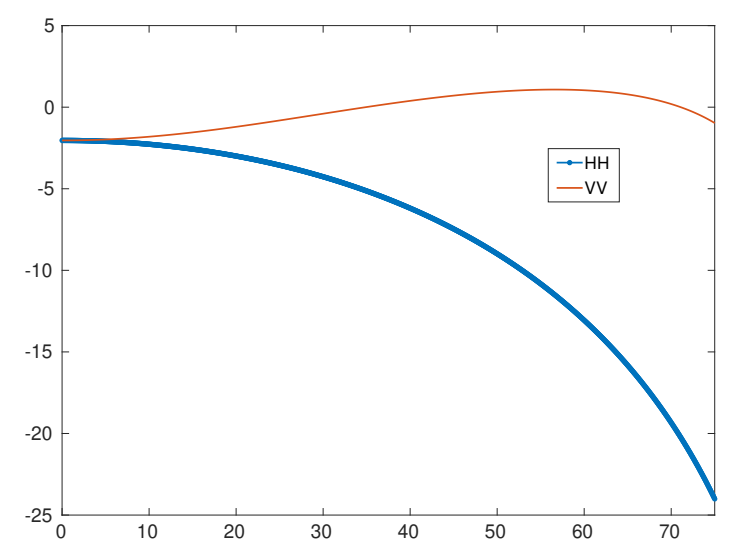

Fig. 3: Geometrical NRCS factor $(\cos \theta)^{4} \mathcal{B}(\theta)$ (in $\mathrm{dB}$ ) as a function of the incidence angle $\theta$ for the two polarizations $\mathrm{VV}$ and $\mathrm{HH}$.

\section{CORRECTING THE POLARIZATION RATIO}

\section{A. Thompson empirical model}

The overestimation of the tilted Bragg model in predicting the PR in microwave bands has been known for long. A very popular model in the literature is the heuristic formulation proposed two decades ago by Thompson et al. [18] for correcting the C- and $\mathrm{Ku}$-band PR:

$$
P R_{T h}=\frac{\left(1+2 \tan ^{2} \theta\right)^{2}}{\left(1+\alpha \tan ^{2} \theta\right)^{2}}
$$

This model depends solely on the incidence angle and a tuning parameter $\alpha$ which is frequency-dependent. With $\alpha=0$, it recovers the Bragg PR in the conducting case (see the kernels in the Appendix):

$$
P R_{\text {Bragg }}=\frac{\left(1+\sin ^{2} \theta\right)^{2}}{\cos ^{4} \theta}
$$

while for $\alpha=1$ it reaches the PR predicted by the Physical Optics (PO) in the conducting case:

$$
P R_{P O}=\frac{1}{\cos ^{4} \theta}
$$

This empirical model was found in very good agreement with experimental PR at medium incidences (20-50 degrees) in C- and $\mathrm{Ku}$-band with estimated values $\alpha=0.6$ and $\alpha=0.95$, respectively. Later on, empirical PR were developed in conjunction with Geophysical Model Functions (GMFs) for describing the observed NRCS in different microwave bands as a function of wind speed, such as the CMOD5 [4] and LMOD [5] GMFs for Cand L-band data, respectively. As many GMFs have been developed from airborne or spaceborne mono-polarized acquisition, empirical PR models were proposed to allow estimation of the NRCS in the other co-polarized channel. By construction these models provide a better representation of the PR. However, they cannot be used to correct the co-polarized NRCS independently from one another and hence cannot be used within the TSM to model the sea clutter distribution.

\section{B. Unified analytical models}

Another approach to improve the PR is relies on a better choice of analytical models to describe rough facet scattering. The essential reason for the failure of the TSM in this respect is that it misses the PO limit, obtained at moderate incidence for very rough seas or high radar frequencies as well as the Geometrical Optics limit obtained in the same conditions at very low incidence. In the PO model, the NRCS is given by a Kirchhoff integral involving the surface autocorrelation function $\rho$ rather than the roughness power spectral density $S$ :

$$
\sigma^{0}(\theta)=16 \pi K_{0}^{4} \cos ^{4} \theta \frac{\mathcal{K}}{q_{z}^{2}} \frac{1}{4 \pi^{2}} \int_{\mathbb{R}^{2}} d \boldsymbol{r} e^{i \boldsymbol{q}_{H} \cdot \boldsymbol{r}} e^{-q_{z}^{2} \rho(\boldsymbol{r})},
$$

where $q_{z}=2 K \cos \theta$ is twice the vertical projection of the incident wave vector and $\mathcal{K}$ is a geometrical kernel referred to as the Kirchhoff kernel, depending only on the incidence angle and sea surface permittivity (but not on the polarization). For small roughness $\left(q_{z} \rho<<1\right)$, the surface integral reduces to the Fourier Transform of the autocorrelation function and reduces the PO NRCS 
to a form similar to Bragg scattering 6 , with a different kernel, however $((\mathcal{K} \neq \mathcal{B})$ :

$$
\sigma^{0}(\theta) \rightarrow 16 \pi K_{0}^{4} \cos ^{4} \theta \mathcal{K} S\left(\boldsymbol{q}_{H}\right)
$$

We have chosen to express the last formula in a form similar to the Bragg NRCS (11). We have therefore singled out the geometrical factor $16 \pi K_{0}^{4} \cos ^{4} \theta$ in the expression of the Kirchhoff kernel, which therefore differs from its customary expression given in the literature.

A wealth of modern analytical models referred to as "universal" (see e.g. [19] for a review) are known to extend the domain of validity of Bragg scattering and to comply to the PO limit in appropriate conditions. Many of these models perform a dynamical transition between the PO and Bragg regime, depending on sea state, incidence and radar frequency. This is usually done through the summation of 2 terms, one being one reference asymptotic model (PO, Bragg or SSA1), the other being a Kirchhoff integral with a roughness-dependent kernel that allows to recover the other asymptotic model in the appropriate limit.

This is for example the case with RCA ([20], [21], LCA [22], WCA ([23], [24], Green's function method [25] which seek to correct a PO NRCS. In virtually all these methods, the corrective kernel is built from a difference between the Kirchhoff and the Bragg kernel, a property which is necessary to ensure the dynamical transition between the Bragg and Kirchhoff regime.

\section{An analytical approach to Thompson empirical model}

The universal analytical methods proceed via an integration of a Kirchhoff integral, which is in general a delicate numerical task and requires the full knowledge of the ocean spectrum. However, in the context of the TSM at medium incidence the calculations should be performed within the local framework of the tilted facets where roughness can be assumed small. Hence, any surface integral of the Kirchhoff is expected to reduce to a term proportional to the wave spectrum at the Bragg resonant frequency. Building on this idea we devise a simple heuristic correction to the TSM Bragg scattering model which captures the essence of a universal scattering model by seeking the rough facet NRCS in the form:

$$
\sigma^{0}(\theta)=16 \pi K_{0}^{4} \cos ^{4} \theta(\mathcal{B}+\beta(\mathcal{K}-\mathcal{B})) S\left(\boldsymbol{q}_{H}\right)
$$

for some dynamical parameter $\beta$ which is expected to be small and depends primarily on sea state and radar frequency. Such a correction is expected to hold at low and moderate incidence only as the corrective term must remain small whereas the difference between the Kirchhoff and Bragg kernels diverges rapidly at large incidences, especially in VV polarization. However, the form of eq. (12) suggests an equivalent empirical correction with a wider angular domain of validity and another parameter $\alpha$ :

$$
\sigma^{0}=16 \pi K_{0}^{4} \cos ^{4} \theta \mathcal{B}\left(1-\xi \alpha \sin ^{2} \theta\right) S\left(\boldsymbol{q}_{H}\right),
$$

with $\xi=1$ in vertical polarization $(\mathrm{VV})$ and $\xi=-1$ in horizontal polarization (HH). This is based on the observation that at low incidence angle:

$$
\frac{\mathcal{K}-\mathcal{B}}{\mathcal{B}} \simeq-\xi A \sin ^{2} \theta
$$

for some $A>0$.

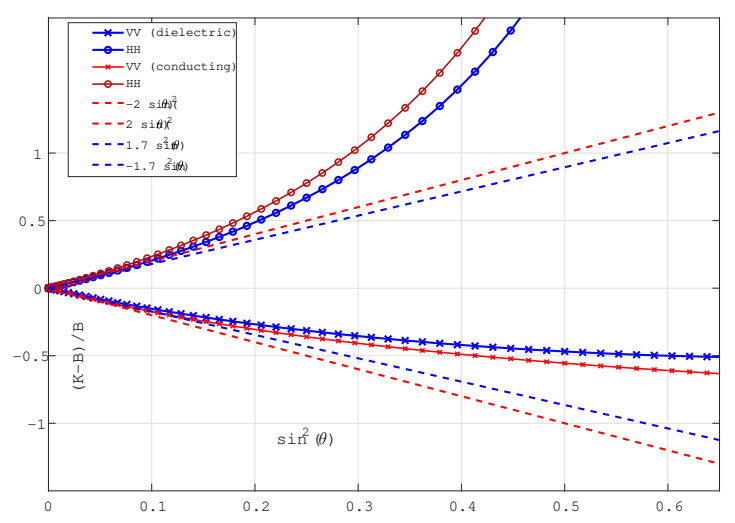

Fig. 4: $(K-B) / B$ versus $\sin ^{2} \theta$

This relation can be easily checked aboth analytically and numerically with a value $A=2$ in the conducting case and $A \simeq 1.7$ for the sea surface permittivity. Figure 4 iilustrates this approximation and confirms its validty at small incidence angle. The additive symetrical correction $\pm A \sin ^{2} \theta$ mitigates the divergence of the left-hand side of eq. (14) in vertical polarization and slightly enhances it in horizontal polarization. Under this simple empirical model the PR is modified to:

$$
P R=(P R)_{\text {Bragg }} \frac{1-\alpha \sin ^{2} \theta}{1+\alpha \sin ^{2} \theta},
$$

where

$$
(P R)_{\text {Bragg }}=\frac{\mathcal{B}_{V V}}{\mathcal{B}_{H H}}
$$

denotes the Bragg PR, where the kernels $\mathcal{B}_{V V}$ and $\mathcal{B}_{H H}$ are recalled in the Appendix. To elucidate the nature of this correction it is interesting to compare the modified PR in the perfectly conducting case with the Thompson's formula (7) for the same values $\alpha$ :

$$
\frac{P R}{P R_{T h}}=\frac{\left(1-\alpha \sin ^{2} \theta\right)\left(1+\alpha \tan ^{2} \theta\right)^{2}}{1+\alpha \sin ^{2} \theta}
$$


Some elementary algebraic manipulations show that:

$$
\frac{P R}{P R_{T h}}=1+\rho(\theta)
$$

where

$\rho(\theta)=\frac{\alpha \sin ^{2} \theta \tan ^{2} \theta}{1+\alpha \sin ^{2} \theta}\left(2+\frac{\alpha\left(1-2 \cos ^{2} \theta\right)}{\cos ^{2} \theta}-\alpha^{2} \tan ^{2} \theta\right)$

is a remainder which remains small for intermediate incidence angles regardless of the value of $\alpha$. Figure 5 shows the evolution of $\rho$ with the incidence angle in the conducting case (where there is a perfect analytical match at small angles) and for the actual sea surface permittivity (taken to be $\epsilon=30+30 i$ ). In this last case, the maximum absolute value of the remainder is 0.1 even though the analytical match at small angle is not perfectly reached. Hence, the proposed correction (15) can be seen as an analytical version of Thompson empirical model.

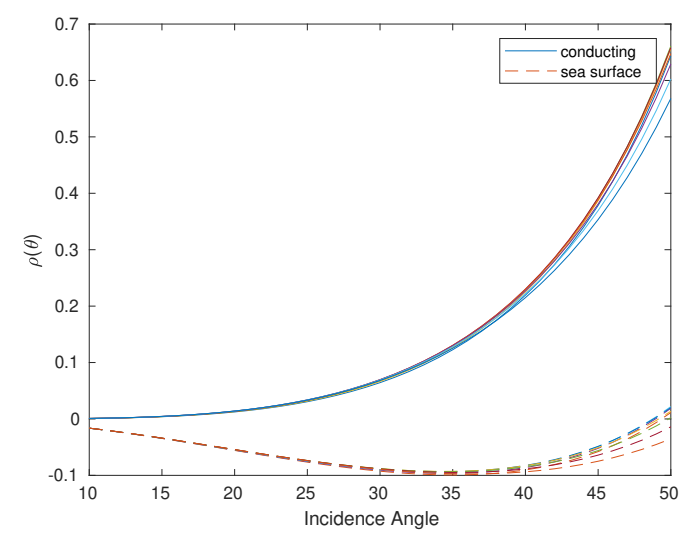

Fig. 5: Evolution of the relative difference $\rho=\left(P R-P R_{T h}\right) / P R$ in equation (18) as a function of the incidence angle for 9 values of the parameter $\alpha$ between 0.6 and 0.95 . The solid lines correspond to the conducting case and the dashed lines to the sea surface dielectric constant.

\section{Experimental evidence of the proposed correction}

The proposed correction (15) for the PR ratio was established on the basis of general theoretical considerations regarding the expected structure of a universal facet model. However, it is also supported by experimental evidence after investigation of some independent data sets. For the relation (15) to hold, the ratio $p(\theta)=$ $P R /(P R)_{B r a g g}$ should satisfy the following relation:

$$
q(\theta)=\frac{1-p(\theta)}{1+p(\theta)}=\alpha \sin ^{2} \theta,
$$

that is the variables $q$ and $\sin ^{2} \theta$ should be linearly related by the coefficient $\alpha$. This observation provides a mean to both check the existence of the relation (15) and evaluate the parameter $\alpha$. This operation was performed with the X-band POLLUPROOF and INGARA airborne datasets, where the full-polarized NRCS was available on a sufficient range of incidence angles for various sea states. Figure 6 shows the function $q(\theta)$ obtained with the X-band POLLUPROOF (wind speed $8 \mathrm{~m} / \mathrm{s}$ ) and INGARA data sets (wind speed $10 \mathrm{~m} / \mathrm{s}$ ). A good linear fitting $q=\alpha \sin ^{2} \theta$ is found for the two data sets with $\alpha=0.77$ and $\alpha=0.89$, respectively. For the INGARA data set, several days of calibrated data were available, with a slight variation of $\alpha$ depending on sea states and wind direction (see Table ??).

\begin{tabular}{|c|c|c|c|c|}
\hline data set & wind (m/s) & dir. & Hs $(\mathrm{m})$ & $\alpha$ \\
\hline POLLUPROOF & 8 & up? & 1 & 0.77 \\
POLLUPROOF & $?$ & $?$ & $?$ & $?$ \\
\hline INGARA & 10.3 & up? & 2.6 & 0.89 \\
INGARA & 9.3 & up? & 2.5 & 0.93 \\
INGARA & 7.90 & up? & 3.5 & 0.80 \\
INGARA & 9.5 & up? & 3.0 & 0.88 \\
\hline
\end{tabular}

TABLE I: Sea states conditions for the X-band airborne measurements POLLUPROOF and INGARA: wind speed (wind), radar aim direction with respect to the wind vector (dir.) and significant wave height (Hs). The value of $\alpha$ is estimated from the best linear fit $q(\theta)=\alpha \sin ^{2} \theta$.
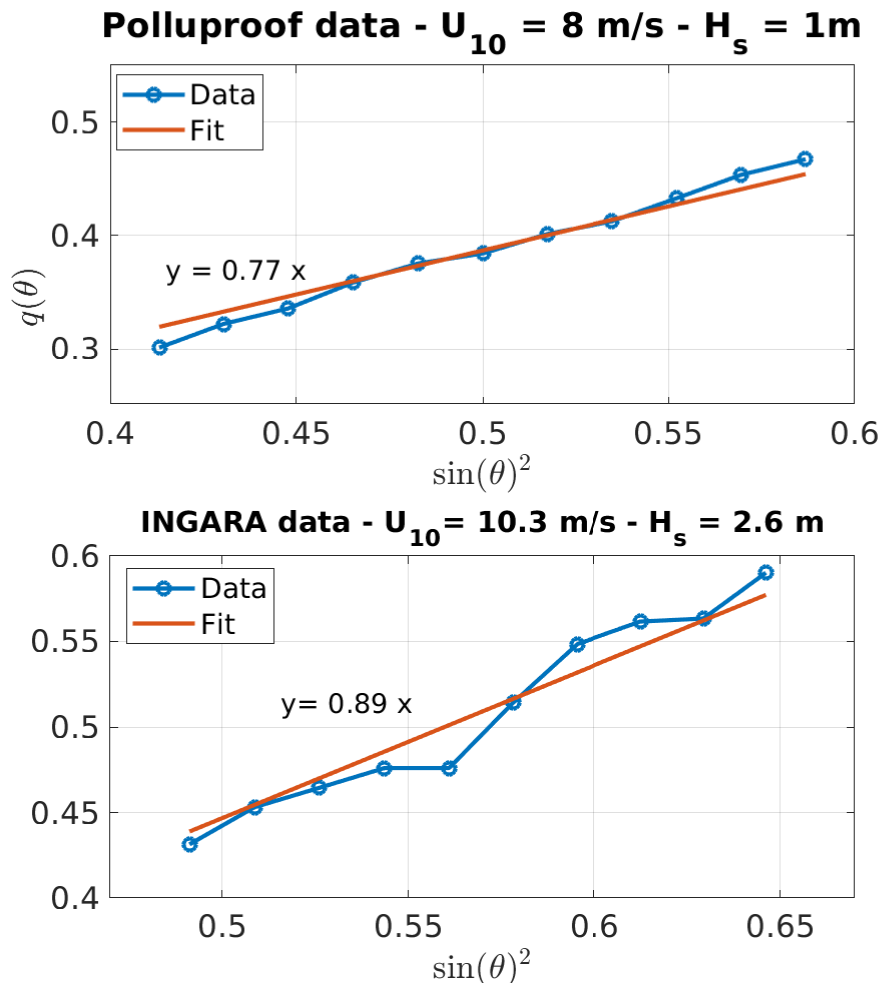

Fig. 6: Experimental evidence of relation (20) in X band. 


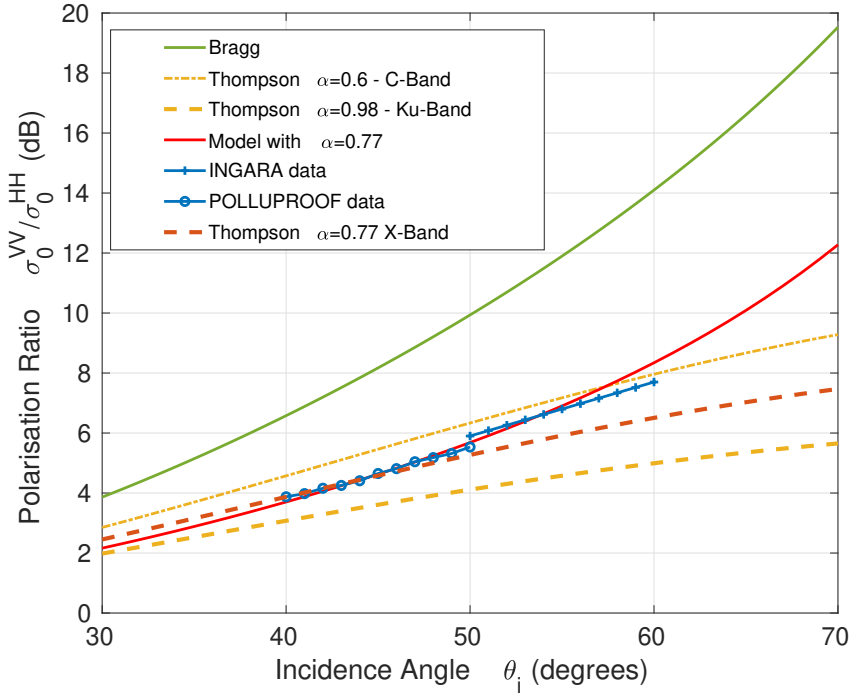

Fig. 7: Corrected PR as a function of incidence in different bands for a moderate wind speed and comparaison with data from the literature
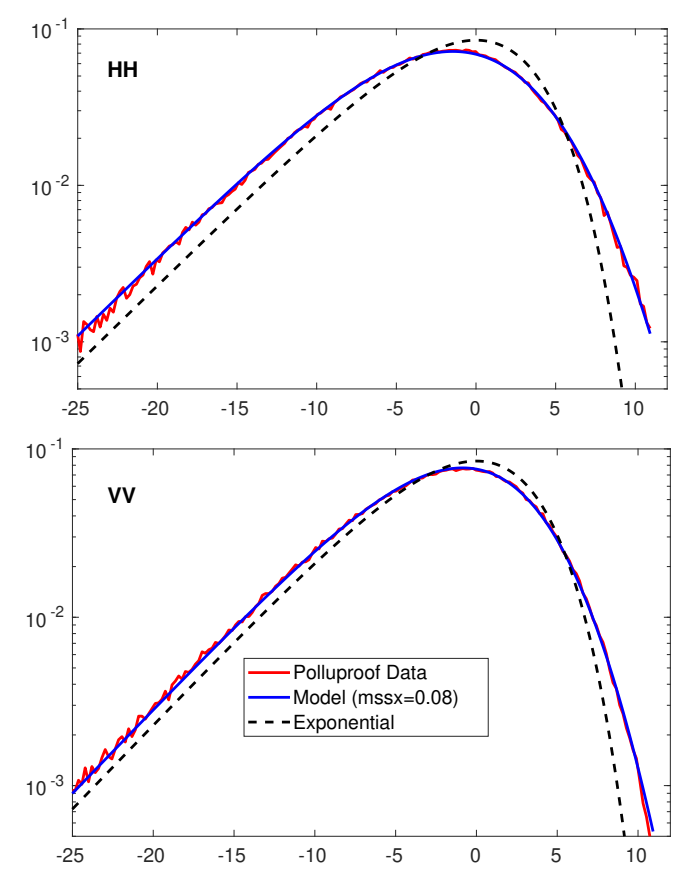

Fig. 8: Intensity distribution with corrected PR $(\alpha=0.009)$ at 45 degree incidence with $m s s_{x}=0.009$.

\section{APPENDiX}

The dielectric Bragg kernels are given by:

$$
\begin{aligned}
& \mathcal{B}_{H H}=\left|\frac{\epsilon-1}{\left(\cos \left(\theta_{i}\right)+\sqrt{\epsilon-\sin ^{2}\left(\theta_{i}\right)}\right)^{2}}\right|^{2} \\
& \mathcal{B}_{V V}=\left|\frac{(\epsilon-1)\left[\epsilon\left(1+\sin ^{2}\left(\theta_{i}\right)\right)-\sin ^{2}\left(\theta_{i}\right)\right]}{\left[\epsilon \cos \left(\theta_{i}\right)+\sqrt{\epsilon-\sin ^{2}\left(\theta_{i}\right)}\right]^{2}}\right|^{2}
\end{aligned}
$$

The dielectric Kirchhoff kernel [26] is given by

$$
\mathcal{K}=\left|\frac{\sqrt{\epsilon}-1}{\sqrt{\epsilon}+1}\right|^{2} \frac{1}{\cos ^{4} \theta}
$$

In the perfectly conducting limit $(\epsilon \rightarrow \infty)$, these kernesl reduce to:

$$
\begin{aligned}
\mathcal{B}_{H H} & =1 \\
\mathcal{B}_{V V} & =\frac{\left(1+\sin ^{2} \theta\right)^{2}}{\cos ^{4} \theta} \\
\mathcal{K} & =\frac{1}{\cos ^{4} \theta}
\end{aligned}
$$

Acknowledgments: We acknowledge the support of the Région Sud for the funding of the first author. The authors are very grateful to L. Rosenberg from the Australian Defence Science and Technology Group for providing the INGARA dataset

\section{REFERENCES}

[1] K. D. Ward, R. J. A. Tough, and S. Watts. Sea clutter: Scattering, the k-distribution and radar performance. 2nd ed. Herts, U.K.: The Institute of Engineering Technology, 2013.

[2] I. Antipov. Statistical analysis of northern australian coastline sea clutter data. Def. Sci. Technol. Org., Canberra, ACT, Australia, Tech. Rep. DSTO-TR-1236, 2001.

[3] Y. Dong. Distribution of $\mathrm{x}$-band high resolution and high grazing angle sea clutter. Def. Sci. Technol. Org., Canberra, ACT, Australia, Res. Rep. DSTO-RR-0316, 2006.

[4] A. Fiche, S. Angelliaume, L. Rosenberg, and A. Khenchaf. Analysis of x-band sar sea-clutter distributions at different grazing angles. IEEE Transactions onGeoscience and Remote Sensing, 53, Aug. 2006.

[5] FM. Farshchian and F. L. Posner. The pareto distribution for low grazing angle and high resolution $\mathrm{x}$-band sea clutter. Proc. IEEE Radar Conf., 2010.

[6] GR Valenzuela and MB Laing. On the statistics of sea clutter. Technical report, NAVAL RESEARCH LAB WASHINGTON DC, 1971.

[7] BL Gotwols and DR Thompson. Ocean microwave backscatter distributions. Journal of Geophysical Research: Oceans, 99(C5):9741-9750, 1994.

[8] Hongkun Li and Joel T Johnson. On the amplitude distributions of bistatic scattered fields from rough surfaces. IEEE Transactions on Geoscience and Remote Sensing, 55(12): $\overline{6883-}$ 6892, 2017.

[9] JW Wright. A new model for sea clutter. IEEE Transactions on antennas and propagation, 16(2):217-223, 1968.

[10] G. Valenzuela. Theories for the interaction of electromagnetic and oceanic waves - a review. Boundary-Layer Meteorology, 13, 1978.

[11] G Soriano and C-A Guérin. A cutoff invariant two-scale model in electromagnetic scattering from sea surfaces. IEEE Transactions on Geoscience and Remote Sensing Letters, 5, 2008.

[12] Stephen O Rice. Reflection of electromagnetic waves from slightly rough surfaces. Communications on pure and applied mathematics, 4(2-3):351-378, 1951

[13] A Voronovich. Small-slope approximation for electromagnetic wave scattering at a rough interface of two dielectric halfspaces. Waves in random media, 4(3):337-368, 1994. 
[14] T. Elfouhaily, B. Chapron, K. Katsaros, and D. Vandemark. A unified directional spectrum for long and short wind-driven waves. J. Geophys. Res., 102, 1997.

[15] D. J. Crisp, N. J. Stacy, and A. S. Goh. Ingara mediumhigh incidence angle polarimetric sea clutter measurements and analysis. Def. Sci. Technol. Org., Canberra, ACT, Australia, Tech. Rep. DSTO-TR-1818, Feb. 2006.

[16] Alexandra Bringer, Bertrand Chapron, Alexis Mouche, and Charles-Antoine Guérin. Revisiting the short-wave spectrum of the sea surface in the light of the weighted curvature approximation. IEEE Transactions on Geoscience and Remote Sensing, 52(1):679-689, 2013.

[17] Tanos Elfouhaily. Physical modeling of electromagnetic backscatter from the ocean surface; Application to retrieval of wind fields and wind stress by remote sensing of the marine atmospheric boundary layer. PhD thesis, Université Paris VII, 1996.

[18] D.R. Thompson, T.M. Elfouhaily, and B. Chapron. Polarization ratio for microwave backscattering from the ocean surface at low to moderate incidence angle. Proceeding IGARSS99, pages 58-67, 2000.

[19] Tanos Mikhael Elfouhaily, Charles-Antoine Guérin, et al. A critical survey of approximate scattering wave theories from random rough surfaces. Waves in Random Media, 14(4):R1R40, 2004.

[20] AA Mouche, B Chapron, and Nicolas Reul. A simplified asymptotic theory for ocean surface electromagnetic wave scattering. Waves in Random and Complex Media, 17(3):321-341, 2007.

[21] Alexis A Mouche, Bertrand Chapron, N Reul, Danièle Hauser, and $\mathrm{Y}$ Quilfen. Importance of the sea surface curvature to interpret the normalized radar cross section. Journal of Geophysical Research: Oceans, 112(C10), 2007.

[22] Tanos Elfouhaily, Stephan Guignard, Ra id Awadallah, and Donald R Thompson. Local and non-local curvature approximation: a new asymptotic theory for wave scattering. Waves in Random Media, 13(4):321-337, 2003.

[23] Charles-Antoine Guérin, Gabriel Soriano, and Tanos Elfouhaily. Weighted curvature approximation: numerical tests for $2 \mathrm{~d}$ dielectric surfaces. Waves in random media, 14(3):349-363, 2004.

[24] Charles-Antoine Guerin, Gabriel Soriano, and Bertrand Chapron. The weighted curvature approximation in scattering from sea surfaces. Waves in Random and Complex Media, 20(3):364-384, 2010.

[25] WT Shaw and AJ Dougan. Half-space green's functions and applications to scattering of electromagnetic waves from oceanlike surfaces. Waves in random media, 5(3):341, 1995.

[26] Alex Stogryn. Electromagnetic scattering from rough, finitely conducting surfaces. Radio Science, 2(4):415-428, 1967. 